\title{
RECONSTRUCCIÓN DEL SISTEMA FONOLÓGICO DEL PROTOCRIOLLO INGLÉS DEL ATLÁNTICO: LAS VOCALES
}

\author{
Mario Portilla
}

\begin{abstract}
RESUMEN
El presente estudio ofrece una reconstrucción del sistema vocálico de la variedad prototípica que dio origen a los diferentes criollos de base inglesa que existen actualmente en el área Atlántica. Este sistema consta de seis vocales largas, seis vocales cortas y tres diptongos. El sistema vocálico reconstruido es bastante semejante al del inglés moderno temprano.
\end{abstract}

\begin{abstract}
The article offers a vowel system reconstruction of the prototypical variety from which the English-based Atlantic Creoles stemmed. The system has six long vowels, six short vowels, and three diphtongs. The reconstructed vowel system is very similar to that of the Early Modern English.
\end{abstract}

\section{Introducción}

\subsection{Estudios previos de reconstrucción histórica de variedades prototípicas}

Smith (1977a, 1977b, 1978, 1980, 1982a, 1982b, 1982c, 1987) ofrece las primeras muestras de aplicación rigurosa del método histórico comparativo en la reconstrucción parcial del sistema fonológico del protocriollo que dio origen a las variedades criollas de Surinam.

Smith, además de aplicar correctamente el método comparativo para obtener sus reconstrucciones, también recurre al uso de materiales escritos de estados anteriores de las variedades comparadas.

Gilman (1978) lleva a cabo una reconstrucción parcial del sistema fonológico y de algunos aspectos de la morfosintaxis de la variedad prototípica antecesora del CI de Jamaica y del wescos.

Alleyne (1980) ofrece el estudio más amplio de reconstrucción histórica de los criollos de base inglesa. El autor compara las siguientes variedades: gullah, CI de Jamaica, CI de Guyana, sranan, djuka, saramaca y krio.

Este estudio presenta, sin embargo, algunas deficiencias metodológicas, que permiten cuestionar la validez de las reconstrucciones prototípicas. 
En primer lugar, los datos sincrónicos ofrecidos por el autor para algunas variedades (gullah, CI de Jamaica y CI de Guyana) no coinciden con las descripciones dadas por otros autores.

Esto ocurre sobre todo en fonología. Así, por ejemplo, el autor anota que "the vowel systems of the Afro-American dialects under consideration are very uniform", y afirma que el gullah, el CI de Jamaica y el CI de Guyana presentan un sistema de siete vocales, igual al del saramaca. Sin embargo, la afirmación de Alleyne no coincide con las descripciones conocidas y aceptadas de estas variedades ${ }^{1}$.

Otro defecto del estudio de Alleyne estriba en que sus reconstrucciones se basan en el presupuesto de que las variedades de Surinam, y especialmente el saramaca, representan las variedades más conservadoras de los criollos ingleses, mientras que los otros criollos comparados son considerados variedades que han sufrido innovaciones por influjo del inglés estándar.

Jamaican and Krio (and Guyanese and Gullah) are exceptional among the Afro-American dialects (and are becoming more and more so). In these evolved dialects, there is a reduction in the occurrence of nasalization when compared with the Surinam languages (Alleyne 1980:38).

The phonological systems of Ndjuka and Sranan have remained with the single labial fricative [f], but Saramaccan and other dialects have acquired $/ v /$ in their modern phonemic inventories. In the case of Jamaican, Krio, and Guyanese, /v/ is the result of continuing English influence, and /v/ has replaced / b / as the reflex of English [v] almost completely (Alleyne 1980:61).

Del mismo modo, Alleyne parte del supuesto de que las formas más conservadoras son aquellas que son más afines a las lenguas africanas, debido a la teoría de que las lenguas criollas en general se derivan estructuralmente de las lenguas de sustrato.

Por lo tanto, las protoformas reconstruidas preferentemente por Alleyne son aquellas que son más desviantes del inglés estándar moderno y que son supuestamente más semejantes a las lenguas africanas.

(...) considerable attention is therefore devoted to demostrating the relationship between the reconstructed forms of Afro-American dialects and the common substrate: West African languages (Alleyne 1980:34).

El autor del presente estudio considera que los supuestos de Alleyne sobre el origen y la historia de las variedades en cuestión son bastante discutibles.

Además, la aplicación rigurosa del método comparativo exige que las reconstrucciones no se basen en supuestos de orden extralinguuístico, sino en los datos obtenidos de la comparación.

\subsection{Propósitos del estudio}

El presente estudio ofrece una reconstrucción del sistema vocálico de la variedad prototípica que dio origen a los diferentes criollos de base inglesa que existen actualmente en el área Atlántica.

La reconstrucción se basa en la descripción sincrónica de los sistemas fonológicos de 17 variedades, de las cuales se logró obtener suficiente información confiable. Estas variedades son el gullah, CI de Bahamas, CI de Jamaica, CI de Belice, CI de Nicaragua (o CI de la 
Costa Misquita), CI de San Andrés, CI de Costa Rica, CI de Panamá, CI de Barbados, CI de Antigua, CI de Tobago, CI de Guyana, sranan, saramaca, djuka, krio y wescos ${ }^{2}$.

\section{Las protovocales}

Con base en las correspondencias encontradas en las variedades comparadas se puede reconstruir un protosistema de 6 vocales cortas, 6 vocales largas y 3 diptongos.

Este sistema presenta las siguientes oposiciones en términos de rasgos distintivos: [+ vocal], [- consonante], [ \pm anterior $],[ \pm$ alto $],[ \pm$ bajo] y $[ \pm$ largo (ver cuadro 2.1$)$.

\section{Cuadro 2.1}

\section{Sistema vocálico del protocriollo inglés del Atlántico}

\begin{tabular}{|c|c|c|c|c|}
\hline & \multicolumn{2}{|c|}{$[+$ anterior $]$} & \multicolumn{2}{|c|}{ [- anterior $]$} \\
\hline & [- largo] & [+ largo $]$ & [+ largo] & [- largo] \\
\hline $\begin{array}{l}\text { [+ alto] } \\
{[\text { - alto }}\end{array}$ & I & i: & u: & $v$ \\
\hline - bajo] & $\varepsilon$ & e: & $0:$ & $\wedge$ \\
\hline [+ bajo] & a & $\mathrm{a}:$ & ว: & D \\
\hline
\end{tabular}

Diptongos: $\quad$ aI $\Lambda \mathrm{I} \Lambda \mathrm{U}$

Las protovocales presentan las siguientes correspondencias en las variedades comparadas:

\subsection{Las vocales cortas}

\subsection{1. $*_{I}$}

\section{/ i /}

SRA: bigi grande (big)

físi pez (fish)

físi idem

SAR: bígi idem

DJU: bígí idem

físí idem

KRI: bíg idem

WES: bík idem fí idem

fif idem síki enfermo (sick) $)^{3}$

síki idem

síkí idem

sỉ idem

sík idem

Es importante señalar que la segunda vocal de los ejemplos correspondientes a los criollos de Surinam es una innovación exclusiva de estas variedades. Por medio de esta regla de epéntesis vocálica se evitan las secuencias de una o dos consonantes finales de palabra existentes en el protocriollo inglés del Atlántico (PIA). Esta regla no se aplica, sin embargo, cuando la palabra termina en la protoconsonante $*_{\mathrm{m}}$. 
*drím tambor $>$

SRA: drón idem

SAR: doón idem

DJU: doón idem

/ I /

GUL, BAH, BEL, NIC, SAN, COS, PAN, JAM, BAR, ANT, TOB, GUY: bíg grande fí pez sík enfermo

2.1.2. *v

/ u /

SRA: fútu pie (foot)

SAR: fútu idem

DJU: fútú idem

fúru lleno (full)

fúu idem

búku idem

KRI, WES:

fút idem

fúú idem

búkú idem

fúl idem búk idem

/ / /

GUL, BAH, BEL, NIC, SAN, COS, PAN, JAM, BAR, ANT, TOB, GUY: fひt pie

fひ̛t lleno bびk libro

2.1.3. $* \varepsilon$

/ e /

SRA: déde morir (dead)

DJU: dédé idem

WES:

ébi pesado (heavy)

hébí idem

hévi idem

ébi pesado bédi cama (bed)

bédí idem

bét idem

/ $\varepsilon / \mathrm{y} / \mathrm{e} /$

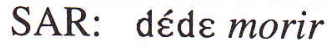

bédi cama

En saramaca, los reflejos de la protovocal $* \varepsilon$ parecen haberse fusionado con los reflejos de la protovocal *e: (cf. apartado 2.2.3) en un estadio primitivo de lo que ha sido denominado protocriollo de Surinam (PCS) ${ }^{4}$. La fusión de ambas protovocales en este primer estadio del protocriollo de Surinam puede ser representado por medio de la siguiente regla:

Regla 2.1.

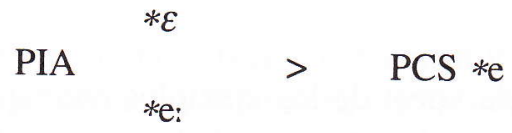

Los siguientes ejemplos muestran cómo las protovocales $* \varepsilon$ y $*$ del PIA no se corresponden con las vocales / $\varepsilon / \mathrm{y} / \mathrm{e} / \mathrm{del}$ saramaca moderno. 


$$
\begin{array}{lll}
* \varepsilon & >\quad & \mathrm{d} \varepsilon \mathrm{d} \varepsilon \text { morir }(\text { dead }) \\
& & \text { édi cabeza }(\text { head }) \\
* \mathrm{e} . . . \quad>\quad & \mathrm{p} \varepsilon \dot{\varepsilon} \text { jugar }(\text { play) } \\
& \text { sindéki culebra }(\text { snake })^{5}
\end{array}
$$

En un segundo estadio, exclusivo del saramaca, la vocal PCS *e volvió a escindirse a causa de una regla de armonía vocálica que permite predecir la tensión de todas las vocales medias / $\varepsilon, \circ$, e, o / en esta variedad (regla 2.2).

\section{Regla 2.2.}

[+ vocal ]

[- consonante ]

[ - alto ]

[ - bajo ] $[\alpha$ tenso $] \quad / \quad-(\mathrm{C}) \quad[-$ consonante $]$

[ $\alpha$ tenso ]

Según esta regla, la vocal floja / $\varepsilon$ / ocurre delante de una sílaba que contiene una vocal también media floja, mientras que la vocal tensa / e / aparece delante de una sílaba que contiene una vocal tensa ${ }^{6}$.

Ejemplos de la regla anterior en reflejos de la protovocal $* \varepsilon$ son los siguientes:

sében siete (seven)

suméc oler (smell)

édi cabeza (head)

eépi ayudar (help)

Por último, en esta variedad, las palabras monosilábicas que contienen una vocal media presentan la vocal / $\varepsilon$ / en posición final y la vocal / e / delante de consonante nasal.

Regla 2.3.

$\begin{array}{llll}\mathrm{PSC} * \mathrm{e}> & \varepsilon & / & -\# \# \\ & \mathrm{e} & / & \mathrm{N}\end{array}$

Ejemplos de la regla anterior en reflejos de las protovocales * $\varepsilon$ y *e son los siguientes:

wE bien (well)

sén vergüenza (shame $)^{7}$

$/ \varepsilon \sim \mathrm{e} /$

KRI:

Ébi pesado

béd cama

ébi

béd

Otros ejemplos de la variación entre estos fonemas son los siguientes:

$\varepsilon$ d éd cabeza (head)

Ég ég huevo (egg) 
En krio la variación en los reflejos de la protovocal $* \varepsilon$ puede ser explicada como un caso de cambio en proceso. Se supone que en esta variedad coexisten, pues, varios sistemas fonológicos diferentes. Por un lado, en un sistema máximo de oposiciones fonológicas, la protovocal ${ }^{*} \varepsilon$ se realiza como el fonema / $\varepsilon /$. Este fonema se opondría consistentemente a un fone$\mathrm{ma} / \mathrm{e} /$, el cual se deriva de otra protovocal (cf. 2.2.3). Por otro lado, en un sistema mínimo de oposiciones, la protovocal * $\varepsilon$ se realiza como el fonema / e /, fundiéndose, por lo tanto, con el fonema ya existente / e /. Este proceso puede ser representado de la siguiente forma:

\section{Cuadro 2.2}

\section{Reflejos de los protofonemas ${ }^{*} \varepsilon$ y $*$ e: en krio}

Protovocales

Sistema Máximo

Sistema Mínino

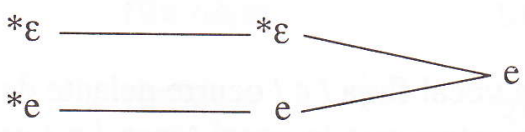

$/ \varepsilon /$

GUL, BAH, BEL, NIC, SAN, COS, PAN, JAM, BAR, ANT, TOB, GUY:

$\begin{array}{lll}\text { déd morir } & \text { (h) Évl pesado } & \text { (h) } \varepsilon \text { bl }\end{array}$

2.1.4. $*_{\Lambda}$

/ o /

SRA: tóno lengua (tongue)

DJU: tóngo idem

són sol (sun)

kóti cortar (cut)

/o/y $/ 0 /$

SAR: tóngo lengua

sónu idem

kóti idem

Al igual que se mencionó para el caso de la protovocal $* \varepsilon$, la protovocal $*_{\Lambda}$ se fusionó con la protovocal *o: (cf. apartado 2.2.4) en un estadio correspondiente al protocriollo de Surinam (PCS).

Regla 2.4.

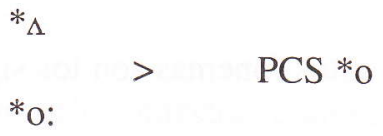


Los siguientes ejemplos muestran cómo las protovocales $*_{\Delta}$ y $*_{0}$ : del PIA no se corresponden con las vocales / o / y / o / del saramaca moderno.

\begin{tabular}{|c|c|c|c|}
\hline$*_{\Lambda}$ & $>$ & $\begin{array}{l}\text { kóndé } \\
\text { diómbo }\end{array}$ & $\begin{array}{l}\text { pueblo (country) } \\
\text { brincar (jump) }\end{array}$ \\
\hline & $>$ & $\begin{array}{l}\text { tádo } \\
\text { bóto }\end{array}$ & $\begin{array}{l}\text { sapo (toad) } \\
\text { bote }(\text { boat })^{8}\end{array}$ \\
\hline
\end{tabular}

Igualmente, en un estadio posterior la vocal PCS *o volvió a escindirse en las vocales $/ \mathrm{o} / \mathrm{y} / \mathrm{o} /$, de acuerdo con las reglas de armonía vocálica de las vocales medias mencionadas en el apartado anterior (regla 2.2).

Ejemplos de esta regla para la protovocal $*_{\Lambda}$ son los siguientes:

$$
\begin{aligned}
& \text { tóng lengua (tongue) } \\
& \text { óndo cien (hundred) } \\
& \text { góni pistola (gun) } \\
& \text { bóbi teta (bubby) }
\end{aligned}
$$

Por último, al igual que en el caso anterior, las palabras monosilábicas que contienen una vocal media presentan la vocal / 2 / en posición final y la vocal / o / delante de consonante nasal.

Regla 2.5.

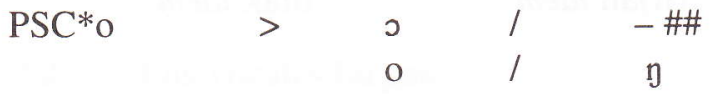

Ejemplos de la regla anterior en reflejos de las protovocales $*_{\Delta} \mathrm{y} *_{0}$ : son los siguientes:

fs cuatro (four)

b́́ $\operatorname{arco}($ bow)

ko venir (come) (PCS *kón, cf. SRA kón y DJU !kon)

/ $\sim \sim \mathrm{a} /$

KRI, WES:

$\begin{array}{lll}\text { kóm venir } & \text { són sol kśt cortar } \\ \text { kám } & \text { sán } & \end{array}$

En estas dos variedades la protovocal $/ *_{\Lambda} /$ tiene dos posibles reflejos. Este hecho puede ser explicado como una coexistencia de sistemas fonológicos en las variedades. Por un lado, en un sistema máximo de oposiciones fonológicas, la protovocal / $*_{\Lambda} /$ se realiza como el fonema $/ \mathrm{o} /$. Este fonema se opondría consistentemente a un fonema / a /, el cual se deriva de otras protovocales (cf. v. gr. 2.1.5). Por otro lado, en un sistema mínimo de oposiciones, la protovocal / $*_{\Lambda} /$ se realiza como el fonema / a /, fundiéndose, por lo tanto, con el fonema ya existente / a /. Este proceso puede ser representado de la siguiente forma: 


\section{Cuadro 2.3}

\section{Reflejos de los protofonemas $*_{\Delta} \mathbf{y} *$ a en krio y wescos}

Protovocales

Sistema Máximo

Sistema Mínino

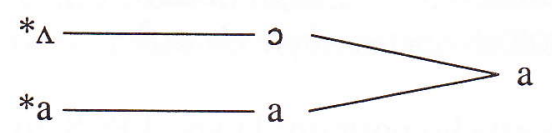

$\mid \Lambda /$

GUL, BAH, BEL, NIC, SAN, COS, PAN, JAM, BAR, ANT, TOB, GUY:

$\mathrm{k}$ m venir SÁn sol kńt cortar

2.1.5. *a

/ a /

SRA: fátu gordo (fat)

SAR: fátu idem

DJU: fátú idem

ánu mano (hand)

-

ána idem

bláka negro (black) baáka idem

baáká idem

KRI, WES, GUL, BAH, BEL, NIC, SAN, COS, PAN, JAM, BAR, ANT, TOB, GUY:

$$
\text { fát idem }
$$

(h)án idem

blák idem

\subsection{6. $*_{\mathrm{D}}$}

/ a /

SRA: bátra botella (bottle)

SAR: báta idem

DJU: batáá idem

náki golpear (nock) ati caliente (hot)

náki idem

nákí idem

BEL, NIC, SAN, COS, JAM, ANT:

bákl idem

nák idem

(h)át idem

TOB, GUY:

bátl idem

nák idem

hát idem

/ o a /

KRI: bstul botella

nák golpear

nók

St caliente

WES: bstru idem

nák idem

hót idem

nók 
Como se ha dicho anteriormente, la variación en los reflejos de los protofonemas en krio y wescos puede ser explicada como una coexistencia de sistemas fonológicos. En el sistema máximo de oposiciones fonológicas, la vocal / o /, como reflejo de los protofonemas $*_{\mathrm{D}}$ y $*_{\Lambda}$, se opone siempre a la vocal / a /, como reflejo de la protovocal *a. En el sistema mínimo de oposiciones, la vocal / o / se fusiona con la vocal / a /.

\section{Cuadro 2.4}

\section{Reflejos de los protofonemas $*_{\mathrm{b}}, *_{\Lambda} \mathrm{y} *_{\mathrm{a}}$ en krio}

Protovocales

Sistema Máximo

Sistema Mínino

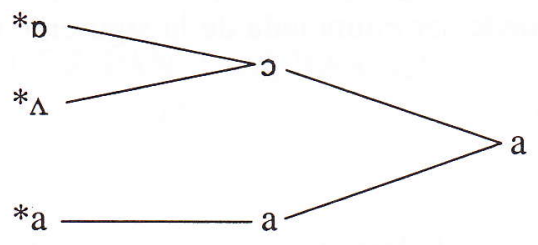

$/ \mathrm{D} /$

GUL, BAH, PAN, BAR, y mesolectos de BEL, NIC, SAN, COS, JAM, ANT, GUY: bótl botella nók golpear hót caliente

\subsection{Las vocales largas}

2.2.1. $*_{i}$ :

\section{/ i /}

SRA: míti encontrar (meet)

SAR: míti idem

siri semilla (seed)

sríbi dormir (sleep)

DJU: mítí idem

síi idem

KRI: mít idem

síi idem

siíbí idem

WES: mít idem

síd idem

slíp idem

-

slíp idem

silíp idem

GUL, TOB:

mit idem

sid idem

slip idem

/ i: / y / I /

ANT: mist encontrar

si:d semilla

slip dormir

En el CI de Antigua, la escisión de la protovocal *i: está condicionada por la posición final de palabra, la cual determina la pérdida del alargamiento de la vocal y, por lo tanto, su fusión con la vocal corta / I /: 
Regla 2.6.

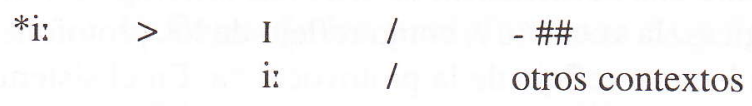

Ejemplos de la regla anterior son los siguientes:

tri árbol (tree)

i:t comer (eat)

dritm soñar (dream) $)^{10}$

La regla anterior es una regla general que se ha aplicado, en realidad, a todas las vocales largas, por lo tanto puede ser enunciada de la siguiente manera ${ }^{11}$ :

Regla 2.7.

$[+$ vocal ]

$[$ - consonante $]>\quad[-$ largo $] \quad / \quad-\# \#$

[+ $\operatorname{largo}]$

/ i: /

BAH, BEL, NIC, SAN, COS, PAN, JAM, BAR, GUY:

mirt encontrar si:d semilla slip dormir

2.2.2. *u:

/ u /

SRA: tú dos (two)

SAR: tú idem

DJU: !tú idem

KRI: tú idem

WES: tú idem

GUL, TOB:

tu idem

lútu raíz (root)

lútu idem

lútú idem

rút idem

úku anzuelo (hook)

húku idem

-

rut idem

úk idem

húk idem

huk idem

/ u: / y / $/$

ANT: $\mathrm{t} d o s$

rust raíz

(h)u:k anzuelo

Al igual que se mencionó en el apartado anterior, en esta variedad la pérdida del alargamiento de la protovocal *u y la consecuente fusión con la vocal corta / / están condicionadas por la posición final de palabra (cf. regla 2.7). 
Regla 2.8.

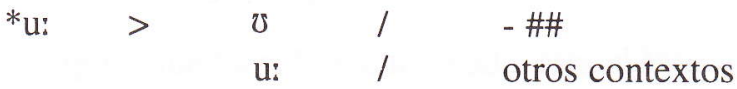

Ejemplos de la regla anterior son los siguientes:

hæ quién (who)

næ nuevo (new)

huifa de quién (who for)

fu:d comida $(\text { food })^{12}$

/ u: /

BAH, BEL, NIC, SAN, COS, PAN, JAM, BAR, GUY:
tu: dos
ruit raíz
(h)u:k anzuelo

2.2.3. *e:

/ e / y / ei ai /

SRA: snéki culebra (snake) nén nombre (name) préi jugar (play)

En sranan la realización de la protovocal *e: como los diptongos / ei / y / ai / está condicionada por la posición final de palabra:

Regla 2.9.

$\begin{array}{llll}* \mathrm{e}: & > & \text { ei } \sim \text { ai } / & -\# \# \\ & \text { e } & / & \text { otros contextos }\end{array}$

Otros ejemplos de la regla anterior son los siguientes:

déi dia (day)

péi pagar (pay)

fési cara (face)

tére cola (tail) $^{13}$

/ e / y / E /

SAR: sindéki culebra

nह nombre

peE jugar

Tal como se ha descrito en el apartado 2.1..3, las protovocales $*^{*}$ y ${ }^{*} \varepsilon$ se fusionaron en un sola vocal en el protocriollo de Surinam (cf. regla 2.1). Luego, esta vocal se volvió a escindir en un estado posterior de este protocriollo para dar origen a las vocales $\varepsilon$ y e en saramaca (cf. reglas 2.2 y 2.3.).

Ejemplos de las reglas anteriores en reflejos de la provocal *e: son los siguientes:

fEE⿱E miedo (afraid)

ne nombre (name)

jési oreja (ear)

jéi oír (hear) ${ }^{14}$ 
/ e /

DJU: sinéki culebra !nen nombre pée jugar

GUL, TOB:

snék idem ném idem plé idem

/ e / y / ia /

KRI, WES:

snék idem ném idem plé idem

En estas variedades, la secuencia de la protovocal *e: más la protoconsonante $*_{r}$ en posición final de palabra se realiza como la secuencia / ia /. Esta regla puede ser expresada de la siguiente manera:

Regla 2.10.

$*$ e: $\mathrm{r} \quad$ ia $\quad / \quad-\#$

Ejemplos de la regla anterior son los siguientes:

ía año (year)

bía cerveza (beer)

antbía oso hormiguero (ant bear)

día caro (dear $)^{15}$

/ ie /

SAN, COS, JAM:

sníek culebra níem nombre plíe jugar

/ ia y $\varepsilon /$

ANT: s(i)niak culebra niam nombre plejugar

La pérdida del alargamiento de la protovocal *e: y la consecuente fusión con la vocal corta / $\varepsilon$ / están condicionadas por la posición final de palabra (cf. regla 2.7).

Regla 2.11.

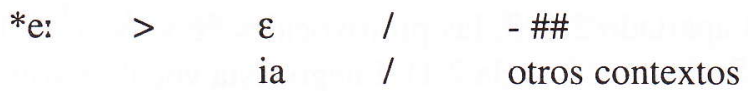

Otros ejemplos de la regla anterior son los siguientes:

pre rezar (pray)

be bahia (bay)

friad temer (fear)

hiar oír (hear) ${ }^{16}$ 
/ e: / y / ea /

BAH: sne:k culebra ne:m nombre ple:jugar

Al igual que ha sido mencionado para el krio y el wescos, en esta variedad la secuencia de la protovocal *e: más la protoconsonante *r en posición final de palabra se realizan como la secuencia / ea /.

Regla 2.12.

*er $>$ ea $/ \quad-\#$

Un ejemplo de la regla anterior es el siguiente:

hea oir (hear) $)^{17}$

/ e: /

BEL, NIC, PAN, BAR, GUY y variedades mesolectales de SAN, COS, JAM, ANT: sne:k culebra ne:m nombre ple: jugar

2.2.4. $*_{O}$ :

/o /

SRA: stón piedra (stone) bóto bote (boat) dóro puerta (door)

DJU: sitón idem bóto idem dóó idem

GUL, TOB:

ston idem bot idem do idem

/ o / y / al

SAR: sitónu piedra bóto bote dós puerta

Tal como se ha descrito en el apartado 2.1.4, las protovocales $*_{0}$ y $*_{0}$ se fusionaron en un sola vocal en el protocriollo de Surinam (cf. regla 2.4). Luego, esta vocal se volvió a escindir, en un estado posterior de este protocriollo, para dar origen a las vocales $\supset$ y o en saramaca (cf. reglas 2.2 y 2.5 ).

Ejemplos de las reglas anteriores en reflejos de la provocal $*_{\mathrm{O}}$ son los siguientes:

bonu hueso (bone)

boóko quebrar (break)

tśdo sapo (toad)

fó cuatro (four) ${ }^{18}$

/ o / y / o o /

KRI, WES:

$\begin{array}{ll}\text { (s)tón piedra } & \text { bót bote } \\ \text { sitón } & \text { dó puerta (door) }\end{array}$

En estas variedades el reflejo regular de la protovocal *o: es la vocal / o /. Sin embargo, la protovocal ${ }^{*} \mathrm{o}$ ante la protoconsonante ${ }^{*} \mathrm{r}$ en posición final de palabra puede realizarse 
ya sea como la vocal / o /, ya sea como la vocal / o /. La regla anterior puede ser enunciada de la siguiente manera:

Regla 2.13.

$$
*_{\mathrm{O}:} \quad>\quad \mathrm{O} \sim 0, \quad-*_{\mathrm{r}}
$$

Ejemplos de la regla anterior son los siguientes:

fó $\sim$ fó cuatro (four)

dó dó puerta (door $)^{19}$

/ uo /

SAN, COS, JAM:

(s)tuón piedra

búot bote

dúor puerta

/ ua y $\wedge$ /

ANT: stuan piedra buat bote ds puerta

La pérdida del alargamiento de la protovocal ${ }^{*}$ : y la consecuente fusión con la vocal corta / $/$ / están condicionadas por la posición final de palabra.

Regla 2.14.

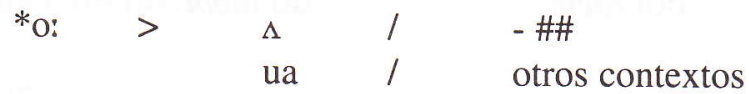

Otros ejemplos son los siguientes:

ual viejo (old)

ruap cuerda (rope)

$\mathrm{n} \Lambda$ saber (know)

slı despacio $(\text { slow })^{20}$

/ O: /

BAH, BEL, NIC, PAN, BAR, GUY y variedades mesolectales de SAN, COS, JAM, ANT: sto:n piedra bo:t bote dor puerta do:

2.2.5. *a:

/ a /

SRA: grási hierba (grass) srápu afilado (sharp) láfu reír (laugh) dánsi bailar (dance)

SAR: gaási idem saápu idem

DJU: gáásí idem saápú idem láfu idem

láfú idem

dánsí idem

KRI, WES, GUL, TOB:

grás idem

Jáp idem

láf idem

dáns idem 
/ a: /

BAH, BEL, SAN, NIC, COS, PAN, JAM, ANT, BAR, GUY:
grá:s hierba
Já:(r)p afilado
lá:f reír
dáns bailar

2.2.6. $*_{\text {ว: }}$

/ a /

SRA: wáka caminar (walk) wátra agua (water) sáfu suave (soft) biká(si) porque (because)

SAR: wáka idem

wáta idem

- biká idem

DJU: wáká idem

watáá idem

sáfú idem .

bikáá idem

$$
\text { / } 2 \sim \mathrm{a} /
$$

KRI, WES:

$\begin{array}{llll}\text { wók(a) caminar } & \text { wotá agua } & \text { sóf suave } & \text { bikós porque } \\ \text { wáka } & \text { watá } & \text { sáf } & \end{array}$

En krio y wescos, en el sistema máximo de oposiciones fonológicas, la vocal / a / refleja los protofonemas $*_{2}$, $*_{0}$ y $*_{\Lambda}$ y se opone siempre a la vocal / a /, la cual refleja las protovocales *a: y*a. En el sistema mínimo de oposiciones, la vocal / $/$ / se fusiona con la vocal / a $/$.

\section{Cuadro 2.5}

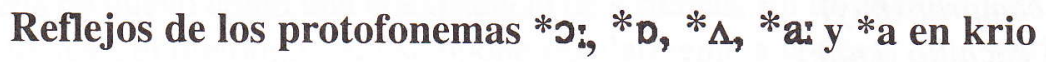

Protovocales

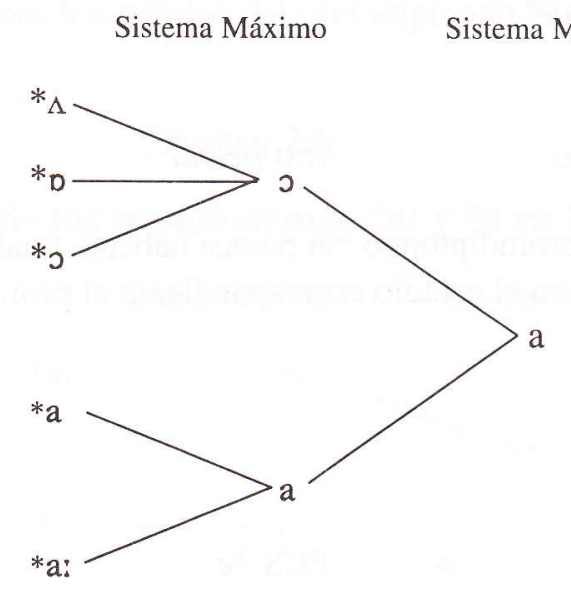

/ o /

GUL: vok caminar

vota agua

spf suave

(bi)koz porque

/ a: /

BEL, NIC, SAN, COS, JAM, ANT ${ }^{21}$, TOB, GUY:

wá:k caminar

wá:ta agua sáf suave

(br)ká:(z) porque 
/ $0: /$

BAH, PAN, BAR y mesolectos de BEL, NIC, SAN, COS, JAM, GUY:

Wo:k caminar wo:ta(r) agua so:f suave biko:Z porque wo:t $\mathrm{r}$

\subsection{Los diptongos}

\subsection{1. *al}

/ e / y / ei ai /

SRA: dréi seco (dry) féti pelear (fight) néti noche (night)

Al igual que en el caso de la protovocal *e, en sranan la realización del protodiptongo *ar como los diptongos / ei / o / ai / está condicionada por la posición final de palabra.

Regla 2.15.

$\begin{array}{lll}* \text { al } & >\quad \text { ei } \sim \text { ai } / & -\# \\ \text { e } & / & \text { otros contextos }\end{array}$

Otros ejemplos de la regla anterior son los siguientes:

fréi volar (fly)

éi alto (high)

ái ojo (eye)

bái comprar (buy)

néfi cuchillo (knife)

béti morder (bite) ${ }^{22}$

/ e / y / $\varepsilon$ /

SAR: dré seco

féti pelear

ndéti noche

En saramaca, el protodiptongo *aI parece haberse fundido también con los reflejos de las protovocales $* \varepsilon$ y $*_{\text {e: }}$ en el estadio correspondiente al protocriollo de Surinam.

Regla 2.16.

$$
* \text { aI }
$$

PIA *E

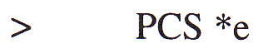

*e:

Como se ha mencionado en el apartado 2.1.3, posteriormente en saramaca esta vocal se volvió a escindir en las vocales / $\varepsilon$ / y / e / debido a una regla general de armonía vocálica, la cual predice la tensión de la vocales medias (cf. regla 2.1.). 
/ e /

DJU: !dree seco

féti pelear

néti noche

/ ai $\sim \varepsilon$ /

KRI: drái seco

fét pelear

náit noche

$n \varepsilon ́ t$

En krio la alternancia entre los reflejos del protodiptongo *aI está condicionada parcialmente por el contexto lingüístico.

Regla 2.17 .

$\begin{array}{llll}* \text { al } & >\quad \text { ai } & / & -\# \\ & \text { ai } \sim \varepsilon & / & \text { otros contextos }\end{array}$

Otros ejemplos de la regla anterior son los siguientes:

flái mosca (fly)

ái alto (high)

ráid réd montar (ride)

ráis rés arroz $(\text { rice })^{23}$

La variación entre el diptongo / ai / y la vocal / $\varepsilon$ / en posición no final de palabra puede ser explicada de nuevo como una coexistencia de sistemas. En un sistema máximo de oposiciones fonológicas, el diptongo / ai / se opone regularmente a la vocal / $\varepsilon$ /, mientras que en el sistema mínimo de oposiciones los reflejos del protodiptongo *aI y de la protovocal * $\varepsilon$ se fusionan en un solo fonema.

\section{Cuadro 2.6}

\section{Reflejos de los protofonemas $*_{a I}$ y $* \varepsilon$ en krio}

Protovocales

Sistema Máximo Sistema Mínino

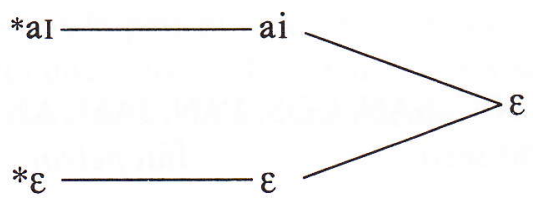

/ ai $\sim$ e /

WES: drái seco

fáit pelear

náit noche (night)

nét

Al igual que en krio, en wescos el protodiptongo *ai presenta dos reflejos. En posición final de palabra ocurre solamente el diptongo / ai /. En otras posiciones el diptongo / ai / y la vocal / e / ocurren como formas alternantes. 
Regla 2.18.

$\begin{array}{llll}* \text { al } & >\quad \text { ai } & / & -\# \# \\ \text { ai } \sim \text { e } / & \text { otros contextos }\end{array}$

Otros ejemplos de la regla anterior son los siguientes:

hái alto (high)

ráid $\sim$ réd montar (ride)

ráis $\sim$ rés arroz (rice)

En este caso, también la variación entre el diptongo / ai / y la vocal / $\varepsilon$ / en posición no final de palabra puede ser explicada como una coexistencia de sistemas. En el sistema máximo de oposiciones fonológicas el diptongo / ai / se opone regularmente a la vocal / e /, mientras que en el sistema mínimo de oposiciones los reflejos del protodiptongo *ai y de las protovocales $* \varepsilon$ y $*$ e se fusionan en un solo fonema.

Cuadro 2.7

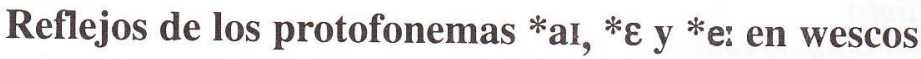

Protovocales Sistema Máximo Sistema Mínino

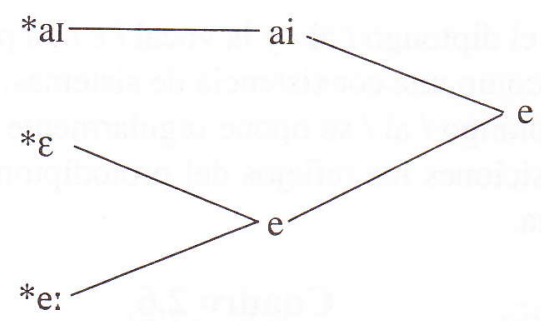

/ $\mathrm{AI} /$

BAR: drúI seco fúit pelear núit noche

/ aI /

GUL, BAH, BEL, NIC, SAN, COS, PAN, JAM, ANT, TOB: drál seco fált pelear náit noche

2.3.3. $/ *_{\Delta l} /$

/ o / y / oi /

SRA: bóri cocinar (boil) póri podrido (spoil) bói muchacho (boy)

En sranan, la realización del protodiptongo $*_{\Delta I}$ como el diptongo / oi / está condicionado por la posición final de palabra: 
Regla 2.19 .

$*_{\mathrm{AI}} \quad>\quad \begin{array}{llc}\text { oi } & / & -\# \# \\ \text { o } & / & \mathrm{C}\end{array}$

SAR: bói idem pói idem

Estas formas en saramaca se derivan presumiblemente de las formas *bóri y *póri en protocriollo de Surinam, tal como se desprende de la comparación con el sranan y el djuka.

DJU: bóli idem

/ oi we /

KRI: bwEl hervir

pwEl estropeado

đ3̧ว in unir

bóil spóil

Las formas alternantes representan probablemente el reflejo de distintas pronunciaciones. La secuencia / we / está condicionada por la presencia de la consonante bilabial que la precede.

Regla 2.20 .

$*_{\text {AI }}>$ we $/ \quad \begin{aligned} & * \mathrm{p}- \\ & * \mathrm{~b}\end{aligned}$

/ $\mathrm{si} /$

WES: bóil hervir

s(i)príl estropeado

đžsin unir

/ aI waI /

COS, JAM, ANT, TOB:
bwáil hervir
(s)pwáil estropeado
báll
spáIl
đzáin unir (join)

Al igual que se ha mencionado para el caso del krio, las formas alternantes representan probablemente diferentes pronunciaciones de la palabra. La secuencia / wa / está condicionada por la presencia de la consonante bilabial precedente.

Regla 2.21 .

$*_{\Lambda I}>$ wa $/ \quad \begin{aligned} & * \mathrm{p}- \\ & * \mathrm{~b}\end{aligned}$

/ aI /

GUL, BEL, NIC, SAN, PAN, GUY:

báil hervir

spáil estropeado

đ亏̌áin unir (join) 
/ $\mathrm{AI} /$

$\mathrm{BAH}, \mathrm{BAR}$ :

ball hervir

spall estropeado

đక̄in unir (join)

/ गI /

Mesolectos de BEL, NIC, SAN, COS, JAM, PAN, GUY:

bóll hervir spóil estropeado

đร̧’́In unir (join)

2.3.4. $*_{\Lambda} \mho$

/ o / y / ow /

SRA: óso casa (house) grón tierra (ground) fówru pájaro (fowl)

En sranan el reflejo más regular del protodiptongo $*_{\Lambda} \mho$ es la vocal / o /. La secuencia / ow / está condicionada por la presencia de la protoconsonante $* 1^{24}$.

Regla 2.22.

$*_{\mathrm{AI}}^{>} \quad \begin{array}{lll}\text { ow } & / & -* 1 \\ 0 & / & \text { otros contextos }\end{array}$

/ (w)o /

SAR: (w)ósu casa goón tierra fóu pájaro

La forma / fóu / se deriva presumiblemente de *fólu, en protocriollo de Surinam, como se desprende de la comparación con el sranan y el djuka. La semivocal / w / protética ante la vocal / o / es una innovación exclusiva del saramaca, cuya aparición está condicionada por la posición inicial de palabra ${ }^{25}$.

Regla 2.23.

$\varnothing \quad>\quad$ w $\quad / \quad \#-0$

$10 /$

DJU: ósu casa

goón tierra

fóo pájaro

/ o au /

KRI, WES:

$\begin{array}{lll}\text { (h)śs casa } & \text { grón tierra } & \text { fól pollo } \\ \text { (h)áus } & \text { graund } & \text { fáwul }\end{array}$

graund

fáwul

En krio, en el sistema máximo de oposiciones, la vocal / o / y el diptongo / au / se oponen entre sí. En el mínimo, la vocal / o / se fusiona con el diptongo / au /. 


\section{Cuadro 2.8}

\section{Reflejos de los protofonemas $*^{*},{ }^{*},{ }^{*} \nu_{;}, y{ }^{*}{ }_{\Delta} \delta$ en krio}

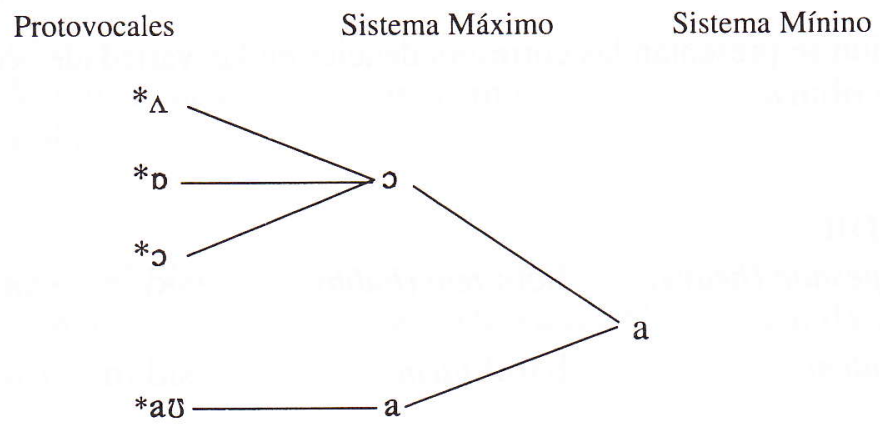

Otros ejemplos de la alternancias entre la vocal / o / y el diptongo / au / son los siguientes:

mót máut boca (mouth)

kó káu vaca (cow)

kónt káunt contar (count)

101

TOB: hós casa grón redondo

\section{/ a /}

GUL, BAH:

(h)árs casa

gráđn tierra

fárl pollo

$/ \Lambda \sim \Delta /$

BEL, NIC, SAN, COS, PAN, JAM, ANT, BAR:

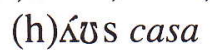

gríßn tierra

grín

fর́ðl pollo

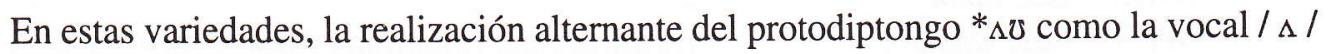
está condicionada por la posición prenasal.

Regla 2.24.

$*_{\Lambda \mho} \quad>\quad \wedge \quad / \quad-\eta$

Otros ejemplos de la regla anterior son los siguientes:

tর́on $\sim$ tín ciudad (town)

díđn dín abajo (down)

bríðn brín café (brown) 


\subsection{Las protovocales en posición inacentuada}

En posición inacentuada en PIA ocurren solamente las siguientes vocales cortas: *I, $* \mho, * \varepsilon, *_{\Lambda} *_{\partial}$.

A continuación se presentan las correspondencias en las variedades comparadas.

2.4.1. $*_{I}$

/ i /

SRA, SAR, DJU:

KRI, WES:

ébi pesado (heavy) bóbi teta (bubby) sidón sentarse (sit-down)

ébi idem

bobi! idem

sidśm idem

/ I /

GUL, BAH, BEL, NIC, SAN, COS, PAN, JAM, ANT, TOB, GUY:

(h)EbI pesado

bubí teta

sidín sentarse

\subsection{2. $*_{\mho}$}

/ u /

SRA: unu ustedes (unu) ${ }^{26}$

SAR: únu idem

KRI: unu idem tumára mañana (tomorrow) pupú idem

/ $/$

BEL, NIC, SAN, COS, PAN, JAM:

ひnび ustedes

tæmára mañana

tromárı

pupú excremento

2.4.3. * $\varepsilon$

/ e /

SRA: bére estómago (belly) -

SAR: bée idem

WES: belé idem hóndret cien (hundred)

$/ \varepsilon /$

SAR: béc estómago (belly)

KRI: bele idem śmdréd cien

GUY: belê idem índred idem

GUL, BEL, NIC, SAN, COS, PAN, JAM:

র́ndred idem

2.4.4. $*_{\Lambda}$

/ O /

SRA: kokronóto coco (coconut) wórom gusano (worm)

DJU: $\quad$ kokoonóto idem wórom idem 
/o /

SRA: kokənóto coco

/ $2 \sim \mathrm{o} /$

KRI, WES:

kokonát coco woróm gusano wíndo ventana (window)

kokonót

$/ \Lambda /$

BEL, COS, PAN, JAM:

kıkanát coco Warśm gusano ${ }^{27}$ wínds ventana

2.4.5. $* 2$

/ a /

SRA: wátra agua (water) úma mujer (woman) kasába yuca (cassava)

SAR: wáta idem

DJU: $\quad$ watáa idem

koósuma idem

umán idem

kasába idem

kasába idem

KRI, WES:

$\begin{array}{lll}\text { watá idem } & \text { úman idem } & \text { kasáva idem } \\ \text { wotá } & \text { wúman } & \text { kasada }\end{array}$

BAH, BEL, NIC, SAN, COS, PAN, JAM, ANT, TOB:

$\begin{array}{lll}\text { wa:tá(r) idem } & \text { ǿman idem } & \text { kasáva idem } \\ \text { wo:ta(r) } & \text { wøman } & \text { kasada }\end{array}$

/ ə /

GUL:

Boto agua

ঔman mujer

kəsavə yuca

kəsadə

$/ \Lambda /$

BAR: wa:tur idem

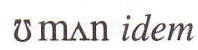

kısavı idem

\section{Algunas reconstrucciones en PIA}

A continuación se presentan algunos ejemplos de palabras reconstruidas con base en la descripción anterior. Por razones de espacio se han elegido solamente algunas de las variedades de las comparadas para mostrar los distintos reflejos de las palabras reconstruidas.

$\begin{array}{llllllllll}\text { PIA } & \text { SRA } & \text { DJU } & \text { KRI } & \text { GUL } & \text { COS } & \text { JAM } & \text { ANT } & \text { TOB } & \\ \text { * béd } & \text { bédi } & \text { bédi } & \text { bẼd } & \text { bed } & \text { béd } & \text { bed } & - & \text { bed } & \text { cama (bed) } \\ \text { *bíg } & \text { bígi } & \text { bígí } & \text { bíg } & \text { bíg } & \text { bíg } & \text { bíg } & \text { bíg } & \text { bíg } & \text { grande(big) }\end{array}$




\begin{tabular}{|c|c|c|c|c|c|c|c|c|c|}
\hline$* b(w)$ & óri & bólí & bwદl & baIl & bwáll & bwáll & bwáll & - & hervir (boil) \\
\hline *dá:ns & dánsi & dánsá & dáns & dans & dá:ns & da:ns & dains & dans & bailar (dance) \\
\hline *dó:g & dágu & dágú & dóg & $\mathrm{dpg}$ & dá:g & da:g & daig & da:g & perro (dog) \\
\hline fff & físi & físí & fís & $\mathrm{ft} \int$ & fif $\int$ & fI $\int$ & $\mathrm{fi} \int$ & $\mathrm{fi} \int$ & pez (fish) \\
\hline$o:(r)$ & fó & !fo & fo/fo & fo & fuor & fuo(r) & fua(r) & 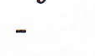 & cuatro (four) \\
\hline$\delta \mathrm{t}$ & fútu & fútú & fút & fost & fớt & fost & fot $t$ & fort & pie (foot) \\
\hline lán & ánu & áná & án & han & án & (h)an & (h)an & han & mano (hand) \\
\hline L & óso & ósú & ós & hars & রỨ & (h) $\Delta \mho \mathrm{s}$ & (h) $\Delta \mho S$ & hos & casa (house) \\
\hline hót & áti & - & 5t & ol & át & (h)at & (h)at & hat & caliente (hot) \\
\hline aít & kóti & kóti & kót & $\mathrm{k} \Delta \mathrm{t}$ & kít & kst & kst & $\mathrm{k} \Delta \mathrm{t}$ & cortar (cut) \\
\hline crál & kréi & eé & krái & krai & král & kraI & kraI & $\mathrm{kraI}$ & llorar (cry) \\
\hline né:m & nén & nén & $\mathrm{em}$ & $\mathrm{em}$ & nfem & nfem & nfam & nem & nombre (name) \\
\hline $10: z$ & nóso & nósú & nós & noz & núoz & núoz & nuaz & - & nariz (nose) \\
\hline nám & nán & !nán & nám & nam & nám & nam & nam & nam & hartar \\
\hline iir & síri & & síc & sid & sí:d & si:d & sitd & id & semilla (seec \\
\hline tú: & tú & & & & & tú: & tひ & tu & dos (two) \\
\hline & wá & wán & wán & - & wán & wan & wan & wan & uno (one) \\
\hline & & itra & watáá & wotá & vota & wá:ta & wa:ta & watta & wa:ta \\
\hline
\end{tabular}

\section{Conclusiones}

El sistema reconstruido de protovocales es bastante simétrico, ya que ofrece una oposición correlativa absoluta entre vocales tensas largas y vocales flojas cortas. El sistema vocálico reconstruido es bastante semejante al sistema del inglés moderno temprano (cf. Prins 1972).

Las variedades de Surinam aparecen como aquellas que han sufrido mayores innovaciones, mientras que las del Caribe parecen ser, en muchos aspectos, más conservadoras.

Es importante señalar también que en muchos aspectos los mesolectos parecen ser aún más conservadores que los basilectos.

En general, la reconstrucción fonológica ofrecida en este trabajo no concuerda con los resultados de la reconstrucción propuesta por Alleyne (1980). Esto se debe, sin duda alguna, a que este autor basa su reconstrucción básicamente en las variedades de Surinam, especialmente en el saramaca.

\section{Lista de abreviaturas}

$\begin{array}{ll}\text { ANT } & \text { Criollo inglés de Antigua } \\ \text { BAH } & \text { Criollo inglés de Bahamas } \\ \text { BAR } & \text { Criollo inglés de Barbados } \\ \text { BEL } & \text { Criollo inglés de Belice } \\ \text { C } & \text { Consonante } \\ \text { CI } & \text { Criollo inglés }\end{array}$




$\begin{array}{ll}\text { COS } & \text { Criollo inglés de Costa Rica } \\ \text { DJU } & \text { Djuka } \\ \text { GUL } & \text { Gullah } \\ \text { GUY } & \text { Criollo inglés de Guyana } \\ \text { JAM } & \text { Criollo inglés de Jamaica } \\ \text { KRI } & \text { Krio } \\ \text { N } & \text { Consonante nasal } \\ \text { NIC } & \text { Criollo inglés de Nicaragua } \\ \text { PAN } & \text { Criollo ingles de Panamá } \\ \text { PCS } & \text { Protocriollo de Surinam } \\ \text { PIA } & \text { Protocriollo inglés del Atlántico } \\ \text { SAN } & \text { Criollo inglés de San Andrés } \\ \text { SAR } & \text { Saramaca } \\ \text { SRA } & \text { Sranan } \\ \text { TOB } & \text { Criollo inglés de Tobago } \\ \text { V } & \text { Vocal } \\ \text { WES } & \text { Wescos }\end{array}$

\section{Notas}

1. Cf. Turner, 1945 y 1949 para el gullah; Cassidy \& LePage, 1967[1980]; Wells, 1982 para el CI de Jamaica; Bickerton, 1975 y Rickford, 1987 para el CI de Guyana.

2. Las fuentes en las que se basa la comparación son las siguientes:

Gullah (Turner, 1945; 1949)

CI de Bahamas (Wells, 1982)

CI de Belice (Datos de trabajo de campo)

CI de Nicaragua (Datos de trabajo de campo)

CI de San Andrés (Datos de trabajo de campo)

CI de Costa Rica (Datos de trabajo de campo)

CI de Panamá (Datos de trabajo de campo)

CI de Jamaica (Wells, 1973)

CI de Barbados (Wells, 1982)

CI de Antigua (Farquhar, 1974)

CI de Tobago (Southers, 1975)

CI de Guyana (Wells, 1982; Rickford, 1987; Carter, 1987)

Sranan (Taylor, 1977; Echteld, 1961)

Saramaca (Taylor, 1977; Voorhoeve, 1959; Rountree, 1972)

Djuka (Huttar \& Huttar, 1972)

Krio (Fyle \& Jones, 1980; Carter, 1989)

Wescos (Dwyer, n.d. [1967]; Carter, 1987; Criper-Friedman, 1990).

3. El símbolo [ ' $]$ representa tono alto para las siguientes variedades: saramaca, djuka, krio, wescos, CI de San Andrés, CI de Costa Rica y CI de Guyana. Este símbolo también ha sido usado para señalar la sílaba acentuada en el caso del sranan y probablemente del CI inglés de Jamaica. La ausencia del símbolo ['] sobre la vocal representa respectivamente o bien tono bajo o bien ausencia de acento sobre la sílaba respectivamente. Para el caso de las demás variedades, no existen estudios adecuados para determinar el estatus fonológico de los suprasegmentales.

Las palabras entre paréntesis corresponden a la etimología inglesa de la palabra, y no a la traducción española. 
4. El término ha sido introducido por Smith (1977a) para designar el antepasado común de los criollos de Surinam.

5. Cf. NIC (variedad que conserva la oposición original) ded morir, hed cabeza, ple: jugar, sne:k culebra.

6. Esta regla de asimilación ocurre tanto con palabras de origen inglés como con palabras de otros orígenes: mujế < portugués / mu' $\measuredangle \varepsilon r /$ mujer

pénti < portugués / "pẽnti / peine

fênse < holandés / "venstər / ventana

kéíki < holandés / 'kerk / iglesia

lébê-lébế < kikongo / lébé-lébé / alto y delgado (Daeleman 1972)

tengú-tengú < kikongo / téngú-téngu / saltar (Daeleman 1972)

7. Cf. NIC wel bien, Jem vergüenza.

8. Cf. NIC kAntrI pueblo, d3Amp brincar, to:d sapo, bo:t bote.

9. Cf. NIC for cuatro, kAmvenir.

El símbolo (!) indica que en djuka los tonos correspondientes de estas palabras no han sido señalados en las fuentes.

10. Cf. COS tri: árbol, i.t comer, drim soñar.

11. Esta regla fonológica diacrónica no es exclusiva del CI de Antigua, sino que existe también en el CI de St. Kitts (cf. Wells 1980:76).

12. Cf. COS hu: quién, nu: nuevo, fu:d comida.

La forma hu:fa proviene presumiblemente del inglés who for.

13. Cf. NIC de: día, pe: pagar, fe:s cara, te:l cola.

14. Cf. NIC fre:d miedo, nem nombre, e:s oreja, herr oír.

15. Cf. NIC err año, berr cerveza, antsber oso hormiguero, derr caro.

16. Cf. NIC pre: rezar, be: bahía, fre:d miedo, herr oir.

17. Cf. NIC herr oír.

18. Cf. NIC born hueso, bro:k quebrar, to:d sapo, for cuatro.

19. Cf. NIC for cuatro, dort puerta.

20. Cf. NIC o:l viejo, ro:p cuerda, no: saber, slo: despacio,

21. Wells (1980) señala que en el CI de Montserrat (Islas de Sotavento) la regla de acortamiento vocálico afecta también a las vocales largas bajas. Desgraciadamente, no fue posible corroborar con los datos disponibles este hecho en el CI de Antigua.

22. Cf. COS fláı volar, áı alto, náif cuchillo, báıt morder.

23. Cf. COS flái volar, áı alto, ráı montar, ráis arroz. 
24. Smith (1982) supone los siguientes estados en la derivación de la palabra en PCS:

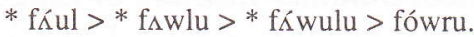

25. Cf. las siguientes palabras de origen portugués:

(w)óbo </'ovo / huevo

(w)ómi </'smẽj/ hombre

26. Esta palabra proviene presumiblemente del igbo (Hancock 1969: 62).

27. Esta forma ha sido recogida solamente para el caso de Jamaica y Panamá.

\section{Bibliografía}

Alleyne, M. 1980. Comparative Afro-American. Ann Arbor: Karoma.

Bickerton, D. 1975. Dynamics of Creole system. Cambridge: Cambridge University Press.

Carter, H. 1987. Suprasegmentals in Guyanese: some African comparisons. En Gilbert (ed.), 213-63.

1989. Three Creole pitch systems. En Haïk \& Tuller (eds.): 27-44.

Cassidy, F. \& R. LePage. 1967 [1980]. Dictionary of Jamaican English. Cambridge: Cambridge University Press.

Criper-Friedman, L. 1990. The tone system of West African English. World Englishes. 9: 63-77.

Daeleman, J. 1972. Kongo elements in Saramacca Tongo. Journal of African Languages. 11: $1-44$.

Dwyer, D. n.d. [1967]. An introduction to West African Pidgin English. Michigan State University.

Echteld, J. 1961. The English words in Sranan. Groningen: Wolters.

Farquhar, B. 1974. A grammar of Antiguan Creole. Tesis doctoral: Cornell University. Fyle, C. \& E. Jones

1980. A Krio-English dictionary. Nueva York: Oxford University Press.

Gilbert, G. (ed.) 1987. Pidgin and Creole languages. Honolulu: University Press of Hawaii.

Gilman, C. 1978. A comparison of Jamaican Creole and Cameroonian Pidgin. English Studies. 59: 57-65. 
Grimes, J. (ed.) 1972. Languages of the Guianas. México: Summer Institute of Linguistics.

Haïk, I. \& L. Tuller (eds.). 1989. Current approaches to African linguistics 6. Dordrecht: Foris Publications.

Hancock, I. 1969. A provisional comparison of the English-based Atlantic Creoles. African. Language Review. 8: 7-72.

Huttar, G \& M. Huttar. 1972. Notes on Djuka phonology. En Grimes (ed.): 1-11.

Prins, A. 1972. A history of English phonemes. Leiden: Leiden University Press.

Rickford, J. 1987. Dimensions of a Creole continuum. Standford: Standford University Press.

Rountree, S. 1972. The phonological structure of stems in Saramaccan. En Grimes (ed.), 1972: 22-7.

Smith, N. 1977 a. Vowel Epithesis in Surinam Creoles. Amsterdam Creole Studies. 1: 1-30.

1977 b. The development of the liquids in the Surinam Creoles. Amsterdam Creole Studies. 1: 31-54.

1978. On the liquefying of / d / in the Surinam Creole languages. Amsterdam Creole Studies. 2: 115-24.

1980. Some nasal phenomena in the Creole languages of Surinam. Amsterdam Creole Studies. 3: 14-24.

1982 a. On the liquefying of / d / in the Surinam Creole languages again. Amsterdam Creole Studies. 4: 104-14.

1982 b. Owroe fowroe kowroe: English or Dutch? Amsterdam Creole Studies. 4: 110-7.

1982 c. The development of nasal-stop clusters in the Surinam Creoles. Amsterdam Creole Studies. 4: 90-103.

1987. The genesis of the Creole languages of Surinam. Tesis doctoral: University of Amsterdam.

Southers, D. 1975. A transformational analysis of Tobagonian Creole English. Tesis doctoral: University of North Carolina.

Taylor, D. 1977. Languages of West Indies. Baltimore: John Hopkins University Press. 
Turner, L. 1945. Notes on the sounds and vocabulary of Gullah. Publication of the American Dialect Society: Number 3. Alabama.

1949. Africanisms in the Gullah dialect. Ann Arbor: University of Michigan Press.

Valdman, A. (ed.). 1977. Pidgin and Creole linguistics. Bloomington: Indiana University Press.

Voorhoeve, J. 1959. An orthography for Saramaccan. Word. 15: 436-45.

Wells, J. 1973. Jamaican Pronunciation in London. Oxford: Blackwell.

1982. Accents of English. Cambridge: Cambridge University Press. 